\title{
A DISPERSIONLESS ALGORITHM FOR CALCULATING WAKE POTENTIALS IN 3D*
}

\author{
R. Hampel**, W.F.O. Müller, T. Weiland \\ Technische Universität Darmstadt, Institut für Theorie Elektromagnetischer Felder (TEMF) \\ Schlossgartenstrasse 8, 64289 Darmstadt, Germany
}

\section{Abstract}

Accurate computations of wake potentials are an important task in modern accelerator design. Short bunches used in high energy particle accelerators excite very highfrequency fields. The geometrical size of accelerating structures exceeds the wavelength of the excited fields by many orders of magnitude. The application of codes such as TBCI, MAFIA[1] or tamBCI are limited due to numerical dispersion effects and memory needs. Recently new codes like PBCI have been developed to overcome these problems. In this work the utilization of dispersionless directions in the leap-frog update scheme on a Cartesian grid are proposed for accurate simulations. In conjunction with a conformal modeling technique which allows for the full Courant time step a moving window technique can be applied. This was previously implemented in a 2D code [2]. In this publication an extension to arbitrary three dimensional problems is presented.

\section{INTRODUCTION}

In this work we present a zero dispersion algorithm in 3D which uses a discretization scheme based on a rotated mesh combined with the SC scheme [3] or the established USC scheme [4] and a moving window technique [5]. The zero dispersion along the beam's propagation direction is combined with the advantages of an explicit algorithm in order to compute wake potentials for long and arbitrarily shaped structures.

Usually a von Neumann analysis is applied to investigate the dispersion properties of a numerical algorithm. Observing a plane wave propagating through a Cartesian mesh allows for finding the numerical dispersion in relation to the propagation direction.

In a Cartesian mesh maximum dispersion occurs along the edges of a mesh cell for the well known leap-frog update scheme. For directions aligned to the diagonals of a mesh cell, i.e. a hexaeder in $3 \mathrm{D}$, the dispersion is found to vanish.

\section{PROPERTIES OF THE ALGORITHM}

The above mentioned dispersion properties of the leapfrog time update scheme on a Cartesian mesh have been implemented so far in the 2D code ROCOCO [2]. The mathematical background for finding these properties is a simple

* This work was partially funded by EUROTeV (RIDS-011899) and DESY Hamburg.

**hampel@temf.tu-darmstadt.de

05 Beam Dynamics and Electromagnetic Fields von Neumann dispersion analysis considering a plane wave propagating through free space. A plane wave propagating along one axis, e.g. the $z$-axis, is described by

$$
W^{(1 D)}(z, t)=A_{0}^{(1 D)} \exp \left(i\left(k_{z} z-\omega t\right)\right) .
$$

Without loss of generality the argument of the exponential function can be generalized to include the propagation of a plane wave in $3 \mathrm{D}$ space

$$
W^{(3 D)}(\vec{r}, t)=A_{0}^{(3 D)} \exp (i(\vec{k} \cdot \vec{r}-\omega t)) .
$$

The dot product $\vec{k} \cdot \vec{r}$ is rotational invariant which allows for introducing spherical coordinates $(k, \phi, \theta)$. Assuming an equidistant mesh the mesh cell becomes a cube and the diagonals of this cube are found to be the dispersionless directions for a propagating wave (Fig. 1).

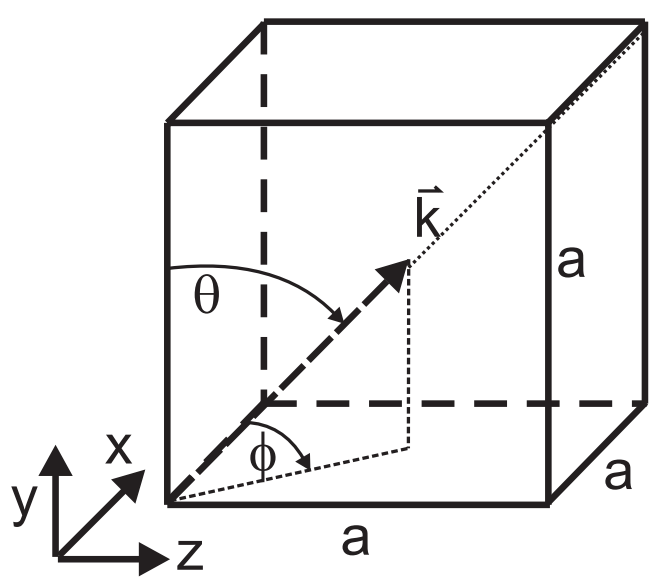

Figure 1: Wave vector $\vec{k}$ of a plane wave propagating through an equidistant mesh cell.

\section{Mesh and Discretization}

Starting from such a rotated mesh cell, the algorithm is realized in a straight forward way. Maxwell's equations for the six field components

$$
\begin{aligned}
& \oint_{\partial A} \vec{E} \cdot d \vec{s}=-\int_{A} \mu \partial_{t} \vec{H} \cdot d \vec{A} \\
& \oint_{\partial A} \vec{B} \cdot d \vec{s}=\int_{A}\left(\epsilon \partial_{t} \vec{E}+\vec{J}\right) \cdot d \vec{A}
\end{aligned}
$$

are discretized on this mesh. A rotation $R$ of the three base vectors $\vec{e}_{x}, \vec{e}_{y}$ and $\vec{e}_{z}$ yields the three new base vectors D05 Code Developments and Simulation Techniques 
$\vec{e}_{u}, \vec{e}_{v}$ and $\vec{e}_{w}$. The rotated mesh cell is shown in Fig. 2 . The diagonal of the cell is aligned to the base vector $\vec{e}_{z}$ of the standard Cartesian mesh.

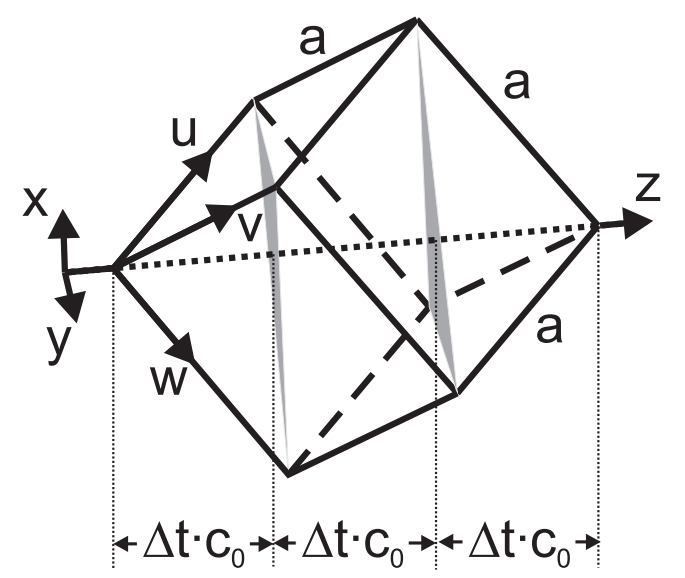

Figure 2: Rotated mesh cell: The diagonal is aligned with the base vector $\vec{e}_{z}$. Three time steps are needed to cross the cell, i.e. beginning of the diagonal $\rightarrow$ gray plane $\rightarrow$ gray plane $\rightarrow$ end of the diagonal.

In order to advance a plane wave or a charge distribution moving at the speed of light $c_{0}$ by one mesh step $\Delta$ the time step is $\Delta t=\Delta / c_{0}$. This time step will generally lead to instabilities except in 1D. The rotation of the mesh enables one to meet the Courant criterion exactly. In the 3D case the time step is $\Delta t=\Delta /\left(c_{0} \cdot \sqrt{3}\right)$.

The same condition is found if the mesh step $\Delta$ is related to the edge length $a \mathrm{x}^{\prime}$ of the mesh cell $\Delta=a / \sqrt{3}$ which leads to a time step of $\Delta t=a /\left(c_{0} \cdot \sqrt{3}\right)$

It should be noted that due to the rotational invariance of Maxwell's equations only the coordinates in the equations are changing - the structure is preserved. The fields and the time update of these fields do not change. One major difference occurs: a more complicated indexing scheme is needed to describe the nodes of the rotated mesh for a subsequent discretization.

\section{Excitation}

In the scope of the work Gaussian shaped bunches are introduced to excite fields in the accelerator structures. Applying currents as an additive term in the equations $1 \mathrm{a}-1 \mathrm{~b}$ leads to a full field formulation. The total field would be involved in the time update scheme. A more comfortable way to implement the excitation is to apply a scattered field approach [6]. The boundary conditions for the total field total $=(s)$ cattered $+(e) x c i t i n g$ are fulfilled on the surface of the structure. Thus separating the scattered and the exciting fields (equations 2a-2b). The exciting fields are determined only once in a preprocessing step. An electrostatic solver is used to find this electric field of a Gaussian shaped bunch propagating on the axis. The exciting fields appear as an additive driving term in equations $2 \mathrm{a}-2 \mathrm{~b}$ and therefore they do not experience numerical dispersion.
Thus, only the scattered fields take part in the time update scheme and noise is reduced.

$$
\begin{aligned}
& \mathrm{d}_{t} \widehat{\mathbf{h}}^{s}=-\mathbf{M}_{\mu^{-1}} \mathbf{C} \mathbf{e}^{s}-\left(\mathbf{M}_{\mu^{-1}}-\mathbf{M}_{\mu_{\mathbf{0}}^{-1}}\right) \mathbf{C} \mathbf{e}^{e}(2 \mathrm{a}) \\
& \mathrm{d}_{t} \mathbf{e}^{s}=\mathbf{M}_{\epsilon^{-1}} \widetilde{\mathbf{C}} \boldsymbol{h}^{s}+\left(\mathbf{M}_{\epsilon^{-1}} \mathbf{M}_{\epsilon_{\mathbf{0}}}-\mathbf{I}\right) \mathrm{d}_{t} \mathbf{e}^{e}(2 \mathrm{~b})
\end{aligned}
$$

Furthermore, in contrast to a current excitation on axis, the excited fields only have to travel from the surface to the axis. This minimizes a source of additional dispersion errors. The discretization of the set of equations $2 a-2 b$ is implemented by using the Finite Integration Technique [7, 8].

\section{Moving Window Technique}

The Gaussian shaped bunches are ultra-relativistic bunches traveling at the speed of light $v=c_{0}$. In terms of causality, no fields can appear in front of the bunch. This allows for the introduction of a moving window technique. Only the region of interest of the wake potential has to be kept in memory. The physical length of structures to be simulated is no longer limited by memory if the moving window is chosen to be of reasonable length.

Finally the structure has to be discretized on the mesh. Usually conformal techniques like PFC $[9,10]$ reduce the time step locally compared to the maximum time step given by the Courant limit. The SC scheme [3] or the USC scheme [4] do not reduce the time step. Both schemes manipulate the entries in a material matrix, i.e. $\mathbf{M}_{\mu^{-1}}$ (USC) or $\mathbf{M}_{\epsilon^{-1}}$ (SC). The assembly of the material matrices and their manipulation can be computed on the fly. No additional memory is needed.

\section{VALIDATION}

The next step is to verify the dispersion properties of the algorithm described above. This is accomplished by propagating a plane wave through free space.

The plane wave is fed into the computational domain on one side propagating along a direction parallel to the mesh cells diagonals. After a certain time the plane wave has traveled into the computational domain. A snapshot of the electric field on the $z$-axis is made and the moving window is activated. After a number of time steps have passed a second snapshot is made. The positions of the two moving windows is shown in Fig. 3.

Since the moving window and the plane wave move at the velocity of light $c_{0}$ there should be no differences in the two fields. The dispersionless direction of the mesh should correspond to a relative permeability of $\mu_{r}=1$ and a relative permittivity of $\epsilon_{r}=1$ in that direction. Thus resulting in $c_{m e s h}=c_{0}$.

The free space is modeled by periodic boundary conditions on the boundaries of the transverse plane of the computational domain.

D05 Code Developments and Simulation Techniques 


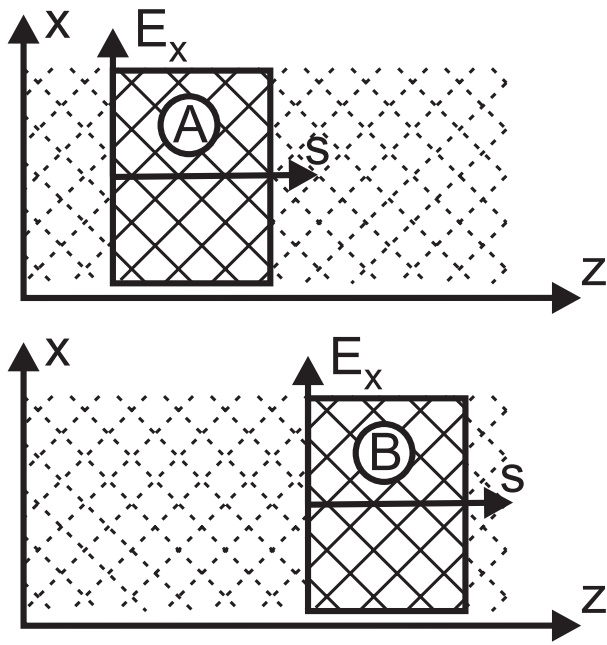

Figure 3: Two moving windows at different times while advancing along the $z$-axis. (A) corresponds to $T=141$. $\Delta t$ and (B) corresponds to $T=237 \cdot \Delta t$ as shown in Fig. 4 and Fig. 5.

In Fig. 4 and Fig. 5 the two snapshots of the electric field $E_{x}(x=0, y=0, t)$ are shown. The propagation direction is towards increasing $z$.

The region of interest is found at positions $s>0$. Both waves show no differences in their shapes within machine accuracy due to the same velocity of the wave and the moving window, i.e. $c_{0}$.

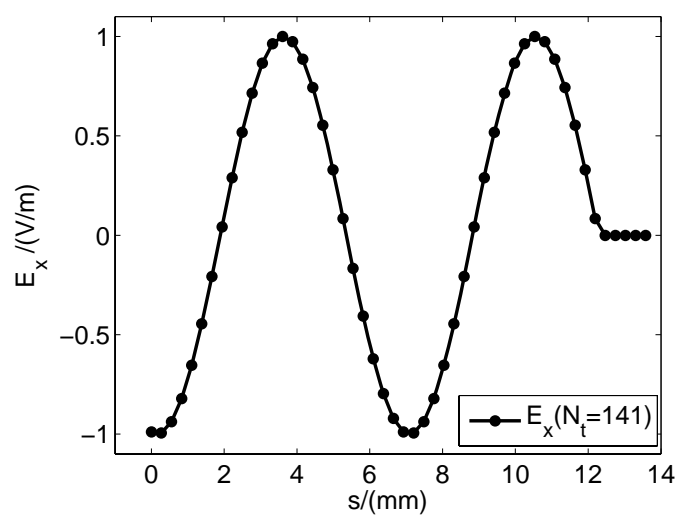

Figure 4: Electric field along the $z$-axis at $T_{1}=141 \cdot \Delta t$. The plane wave and the moving window travel in the positive $z$-direction.

Apparently the two electric fields on the axis have no differences and therefore they travel at $c_{0}$. Due to the space limitations of this paper no fields on other lines parallel to the $z$-axis are shown. But they yield the same result.

\section{CONCLUSION}

In this work we have presented an algorithm which has zero dispersion in given directions, i.e. especially the propagation direction of a wave or a particle bunch. The now 05 Beam Dynamics and Electromagnetic Fields

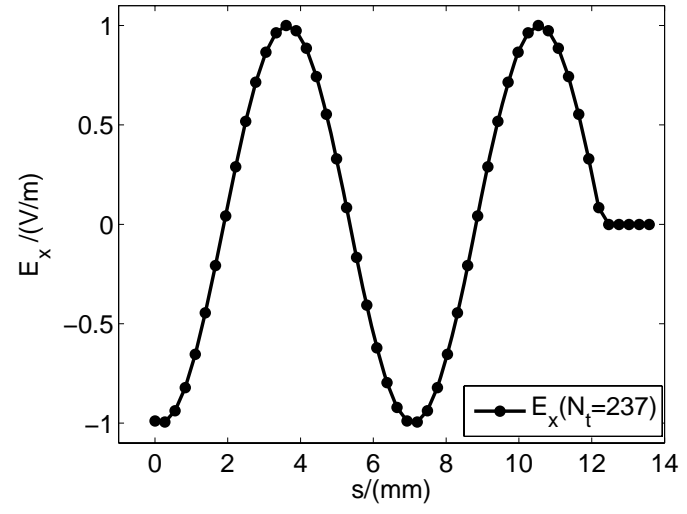

Figure 5: Electric field along the $z$-axis at $T_{2}=237 \cdot \Delta t$. The plane wave and the moving window travel in the positive $z$-direction.

validated algorithm will enable one to compute wake potentials for virtually infinite long accelerator structures.

\section{REFERENCES}

[1] CST GmbH, Bad Nauheimer Str. 19, 64289 Darmstadt

[2] R. Hampel, W.F.O. Müller, T. Weiland, "ROCOCO - A Zero Dispersion Algorithm for Calculating Wake Potentials", presented at the 9th International Computational Accelerator Physics Conference (ICAP), Chamonix, 02.-06.10.2006.

[3] R. Schuhmann, I. Zagorodnov, and T. Weiland, "A Simplified Conformal (SC) Method for Modeling Curved Boundaries in FDTD Without Time Step Reduction", Microwave Symposium Digest 2006, pp. 170-180.

[4] I. Zagorodnov, R. Schuhmann and T. Weiland, "A uniformly stable conformal FDTD-method in Cartesian grids", International Journal of Numerical Modelling, Vol. 16 (2003), pp. 127-141.

[5] K. Bane, T. Weiland, "Wake Force Computation in the Time Domain for Long Structures", Proceedings of the 12th International Conference on High Energy Accelerators, Chicago, 1983, pp. 314-316.

[6] M. Dehler, "Numerical Wake Calculations using an Equivalence Principle", Proceedings of the Particle Accelerators Conference, 1995, Vol. 49, pp. 105-116.

[7] T. Weiland, "A discretization method for the solution of Maxwell's equations for six component fields", Electronics and Communications (AEÜ), Vol. 31 (1977), p. 116.

[8] T. Weiland, "On the Numerical Solution of Maxwell's Equations and Applications in Accelerator Physics", Particle Accelerators Vol. 15 (1984), pp. 245-292.

[9] S. Dey, R. Mittra, "A locally conformal finite-difference time-domain (FDTD) algorithm for modeling threedimensional perfectly conducting objects", IEEE Microwave and Guided Wave Letters 1997; 7(9):273-275.

[10] B. Krietenstein, R. Schuhmann, P. Thoma, T. Weiland, "The Perfect Boundary Approximation Technique Facing the Big Challenge of High Precision Field Computation", Proceedings of the XIX International Linear Accelerator Conference (LINAC 98), Chicago, USA, 1998, pp. 860-862.

D05 Code Developments and Simulation Techniques 